\title{
Common carotid artery dissection caused by radiotherapy: A case report
}

\author{
JIAYAN WANG, DANDAN YUE, XIN CHEN, ZHENYU WEI, WENMEI LU and DANHONG WU \\ Department of Neurology, Shanghai Ninth People's Hospital, \\ Shanghai Jiaotong University School of Medicine, Shanghai 201999, P.R. China
}

Received May 6, 2016; Accepted July 15, 2016

DOI: $10.3892 / \operatorname{mco} .2016 .990$

\begin{abstract}
In the present study, a case of acute cerebral infarction with radiation-induced carotid artery dissection is reported. Carotid artery dissection is generally asymptomatic at the early stages. Due to the non-specific clinical manifestations of carotid artery dissection, a detailed inquiry of the past history of a patient has a critical role in making a diagnosis of radiation-induced common carotid artery dissection. Onset of acute ischemic stroke is the predominant manifestation, and for patients with a history of head-and-neck radiotherapy, dissection should be considered. The condition may progress rapidly, and result in a poor prognosis. Therefore, a correct early diagnosis and initiation of appropriate therapy may lead to rapid recovery, and influence the overall prognosis.
\end{abstract}

\section{Introduction}

As is universally known, radiation therapy can cause numerous side-effects in clinical practice, including side reactions of the respiratory, cerebrovascular and gastrointestinal systems, and so forth. However, carotid artery dissection induced by radiotherapy is infrequent. The present case study reports on the clinical and neuroimaging characteristics of one patient with cerebral infarction caused by radiotherapy.

\section{Case report}

A 66-year-old man was admitted to the emergency department of our hospital (Shanghai Ninth People's Hospital, Shanghai, China) presenting with sudden mild left hemiplegia, hemianesthesia, nausea and vomiting. The patient had a medical history of nasopharyngeal carcinoma treated with radiotherapy

Correspondence to: Dr Danhong Wu, Department of Neurology, Shanghai Ninth People's Hospital, Shanghai Jiaotong University School of Medicine, 280 Mohe Road, Baoshan, Shanghai 201999, P.R. China

E-mail: kathywuxue@sina.com

Key words: nasopharyngeal carcinoma, radiotherapy, common carotid artery dissection, cerebral angiography combined with chemotherapy 10 years previously, and right basal ganglia hemorrhage 1 year prior to his admission. Initially, a computerized axial tomography (PHILIPS Brilliance 64 CT scanner; Philips Healthcare, DA Best, The Netherlands) was performed which revealed no evidence of any high-density lesion (Fig. 1). Intravenous thrombolysis treatment was not administered due to a previous cerebral hemorrhage, although the patient remained within the time window. As the patient's left hemiplegia had deteriorated with the onset of left central facial paralysis, he was transferred to the intensive care unit for monitoring and medical management. A cerebral angiography was performed, with evidence of right common carotid artery dissection and stenosis (Fig. 2). Subsequently, 5,000 U heparin was injected into the patient's body intravenously. With respect to the guide wire and other materials, an $8 \mathrm{~F}$ guided tube was placed in the right common carotid artery, and the protective device was placed at the distal end of the internal carotid artery. A 9x50 mm WALLSTENT ${ }^{\mathrm{TM}}$ stent (Boston Scientific Corporation, Natick, MA, USA) was then imported and released after having been accurately located. Cerebral angiography was subsequently performed, which demonstrated that the dissection and stenosis were markedly improved (Fig. 3). Aspirin (100 mg per day) and clopidogrel (75 mg per day for 1 year) for anti-platelet aggregation, and Lipitor (atorvastatin; $20 \mathrm{mg}$ per day) for stabilizing intravascular plaque, were administered following the stent implantation. The patient is required to continue to take aspirin and Lipitor for life. After the patient was discharged from hospital, all the symptoms went into remission.

\section{Discussion}

Blood flows through the cleft of the carotid artery intima and into the cystic degeneration of the vascular media, thus forming a carotid artery dissection. To the best of our knowledge, carotid artery dissection induced by radiotherapy occurs infrequently. Furthermore, the pathogenesis of radiation-induced carotid artery dissection has yet to be fully elucidated.

Based on a review of the literature, one of the side-effects of radiotherapy is endothelial damage. Little et al (1) proposed that radiation-induced endothelial damage may simply be a consequence of cell loss due to cell killing. In vitro studies have revealed that radiation therapy causes the endothelial colony-forming cells (ECFCs), which are the endothelial 


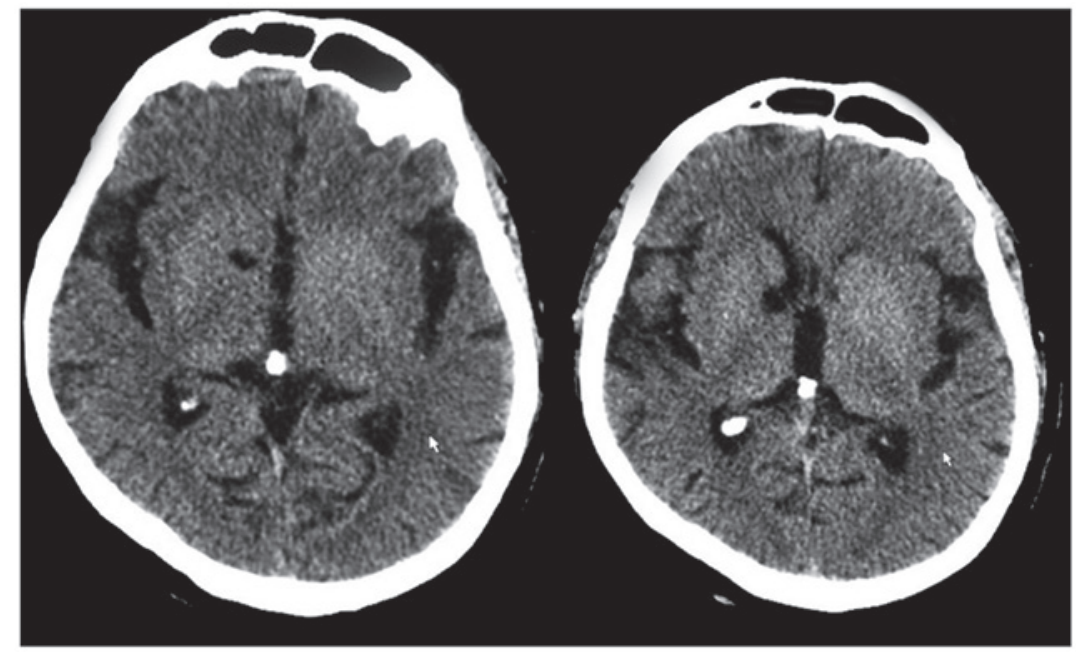

Figure 1. Computerized tomography images, revealing softening lesion at right basal ganglia.

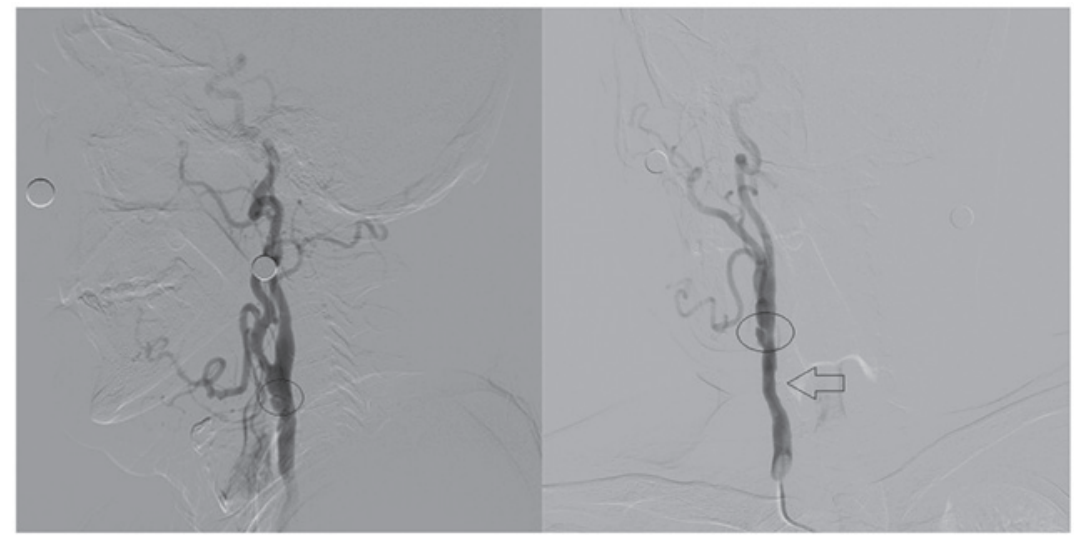

Figure 2. Cerebral angiography images, revealing right common carotid artery dissection (indicated by the circles) and stenosis (indicated by the arrow). The left- and right- hand images show the patient's right common carotid artery from different angles.

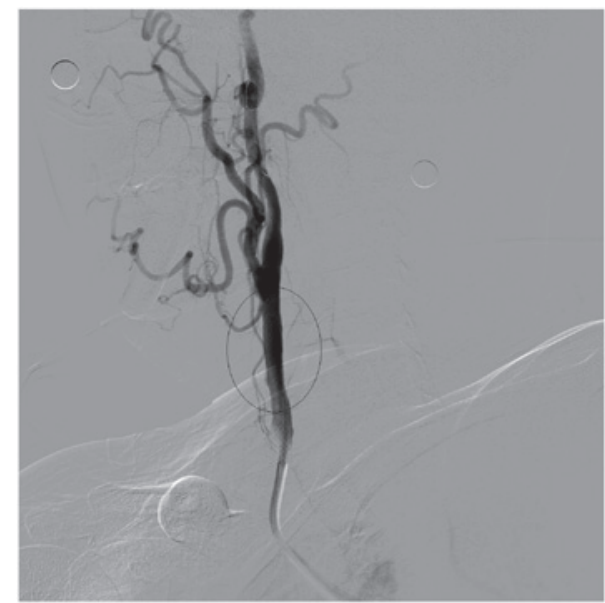

Figure 3. Cerebral angiography image, revealing the marked improvement of the dissection and stenosis (indicated by the black circle).

progenitor cells of the vascular endothelium, to undergo large-scale senescence, which acts as a forerunner of vascular damage and subsequent atherosclerosis (2). Circulating ECFCs have robust proliferative potential and form a profusion of new blood vessels in vivo, thereby exerting an important role in the repair of damaged vascular endothelium $(3,4)$. On the basis of previous research, Pradhan et al (5) discovered that childhood survivors of cancer who received radiotherapy had statistically lower ECFCs and circulating endothelial cells $(\mathrm{P}<0.05)$ compared with those who received no radiotherapy. However, radiation-induced genomic instability, oxidative stress-disrupted mitochondrial function and accelerated cellular senescence have also been implicated in the pathogenesis of endothelial damage and subsequent arteriosclerosis (6-10). Several previous studies demonstrated that radiotherapy of head-and-neck cancer may result in an increase in carotid intima-media thickness and carotid stenosis, consequently leading to a higher risk of cerebrovascular events, including transient ischemic attack and stroke (11-13).

In the present case report, the cause behind the patient's common carotid artery dissection may be that radiotherapy resulted in endothelial damage and the decline of endothelial repair capacity: The damaged parts of his right common carotid artery intima were not able to be repaired quickly, and blood flowed through the cleft of his right common carotid artery intima into the vascular media, thereby forming a carotid 
artery dissection. The dissection is driven by the pressure of blood flow, and gradually expands.

Radiation-induced carotid artery dissection is generally asymptomatic at the early stages. Onset of acute ischemic stroke is the predominant manifestation. For patients with head-and-neck radiotherapy history, dissection should be considered.

In conclusion, the detailed inquiry of a patient's past history has a critical role in making a diagnosis of radiation-induced common carotid artery dissection. This results from the clinical presentation being non-specific. The condition may progress rapidly, and result in a poor prognosis. Therefore, a correct early diagnosis and initiation of appropriate therapy may lead to rapid recovery, influencing the overall prognosis.

\section{Acknowledgements}

The present study was supported by a Research Fund (grant no. 14411972000) of the Science and Technology Commission of Shanghai (to DH.W.), a Research Fund (grant no. 201440332) of the Shanghai Municipal Commission of Health and Family Planning (to DH.W.), and a Research Fund (grant no. YG2013MS23) of Shanghai Jiaotong University (to DH.W.).

\section{References}

1. Little MP, Tawn EJ, Tzoulaki I, Wakeford R, Hildebrandt G, Paris F, Tapio S and Elliott P: Review and meta-analysis of epidemiological associations between low/moderate doses of ionizing radiation and circulatory disease risks and their possible mechanisms. Radiat Environ Biophys 49: 139-153, 2010.

2. Mendonca MS, Chin-Sinex H, Dhaemers R, Mead LE, Yoder MC and Ingram DA: Differential mechanisms of x-ray-induced cell death in human endothelial progenitor cells isolated from cord blood and adults. Radiat Res 176: 208-216, 2011.
3. Ingram DA, Mead LE, Moore DB, Woodard W, Fenoglio A and Yoder MC: Vessel wall-derived endothelial cells rapidly proliferate because they contain a complete hierarchy of endothelial progenitor cells. Blood 105: 2783-2786, 2005.

4. Yoder MC, Mead LE, Prater D, Krier TR, Mroueh KN, Li F, Krasich R, Temm CJ, Prchal JT and Ingram DA: Redefining endothelial progenitor cells via clonal analysis and hematopoietic stem/progenitor cell principals. Blood 109: 1801-1809, 2007.

5. Pradhan K, Mund J, Case J, Gupta S, Liu Z, Gathirua-Mwangi W, McDaniel A, Renbarger J and Champion V: Differences in circulating endothelial progenitor cells among childhood cancer survivors treated with and without radiation. J Hematol Thromb 1: 4, 2015.

6. Schultz-Hector S and Trott KR: Radiation-induced cardiovascular diseases: Is the epidemiologic evidence compatible with the radiobiologic data? Int J Radiat Oncol Biol Phys 67: 10-18, 2007.

7. Andreassi MG and Botto N: DNA damage as a new emerging risk factor in atherosclerosis. Trends Cardiovasc Med 13: 270-275, 2003.

8. Di Lisa F, Kaludercic N, Carpi A, Menabó R and Giorgio M: Mitochondria and vascular pathology. Pharmacol Rep 61: 123-130, 2009.

9. Finsterer J: Is atherosclerosis a mitochondrial disorder? Vasa 36 : 229-240, 2007.

10. Ito TK, Yokoyama M, Yoshida Y, Nojima A, Kassai H, Oishi K, Okada S, Kinoshita D, Kobayashi Y, Fruttiger M, et al: A crucial role for CDC42 in senescence-associated inflammation and atherosclerosis. PLoS One 9: e102186, 2014.

11. Kang HS, Roh JL, Lee SW, Kim SB, Choi SH, Nam SY and Kim SY: Noncancer-related health events and mortality in head and neck cancer patients after definitive radiotherapy: A prospective study. Medicine (Baltimore) 95: e3403, 2016.

12. Gujral DM, Shah BN, Chahal NS, Bhattacharyya S, Hooper J, Senior R, Harrington KJ and Nutting CM: Carotid intima-medial thickness as a marker of radiation-induced carotid atherosclerosis. Radiother Oncol 118: 323-329, 2016.

13. Wilbers J, Meijer FJ, Kappelle AC, Kaanders JH, Boogerd W, Dorresteijn LD, van Dijk EJ and Steens SC: Magnetic resonance imaging of the carotid artery in long-term head and neck cancer survivors treated with radiotherapy. Acta Oncol 54: 1175-1180, 2015. 